\title{
Distributed Feedback-Aided Subspace Concurrent Opportunistic Communications
}

\author{
Jordi Borràs and Gregori Vazquez \\ Department of Signal Theory and Communications, Technical University of Catalonia (UPC) \\ Building D5, Campus Nord UPC, Jordi Girona 1-3, 08034 Barcelona, Spain \\ Email: \{jordi.borras.pino, gregori.vazquez\}@upc.edu
}

\begin{abstract}
This paper deals with the distributed subspace agreement problem for opportunistic communications in time division duplex (TDD) distributed networks. Since scenario-adapted opportunistic transmission schemes rely on locally sampled observations from the wireless environment, degrees-of-freedom (DoF) sensed as available at any node may differ. Transmitting information without agreeing the common active subspace may incur in a performance loss due to noise enhancement, energy loss and inter-system interference. In this context, we propose two subspace concurrence schemes with and without side information about neighboring user's DoF.
\end{abstract}

Index Terms-Opportunistic communications, distributed networks, subspace agreement, feedback, sparse detection.

\section{INTRODUCTION}

Although opportunistic communications [1] permit efficient information transmission in distributed networks, cooperation between opportunistic users leads to an improved overall system performance [2]. Several recent work in the literature address the positive impact of cooperation in terms of spectrum sensing [3], security [4], and scheduling [5]. Summarizing, coordinated communications presents a better performance even in distributed networks.

Authors present in [6] a novel pulse shaping design scheme in which opportunistic nodes exploit only local observations of the wireless environment to design an appropriate opportunistic transmission scheme for time division duplex (TDD). However, as proved therein, there exists an inherent mismatching factor and a signal-to-noise ratio (SNR) loss due to node-to-node uncertainties. In view of the last issue, this paper deals with the problem of subspace concurrence in distributed networks. Basically, we propose two agreement strategies: with and without feedback. In short, the objective of this paper is to identify, in a distributed fashion, the intersection of locallysensed noise subspaces, whereas the uncommon dimensions should be overlooked. Then, once opportunistic nodes use the same subspace, performance loss can be practically avoided.

The problem of subspace consensus is not new. Actually, it has been studied in a wide variety of fields, such as sensor networks [7], automatic control [8], and machine learning [9], among others. Focusing on distributed wireless networks, readers are referred to [10], [11] and references therein.

This research has been funded in part by the Spanish Ministry of Science, Innovation and Universities through project WINTER: TEC2016-76409-C21-R (AEI/FEDER, UE) and fellowship FPI BES-2017-080071, and by the Catalan Government (AGAUR) under grant 2017 SGR 578.
This paper is organized as follows. In Sec. II, we summarize the distributed pulse shaping design scheme presented in [6] and we motivate the interest of subspace concurrence. In Sec. III we formulate the joint waveform detection and subspace dimension reduction. In Sec. IV, we present both non-feedbackaided and feedback-aided subspace intersection identification algorithms. Proposed schemes are numerically assessed in Sec. $\mathrm{V}$, and this paper is concluded in Sec. VI.

\section{PRoblem Definition And Context ReVieW}

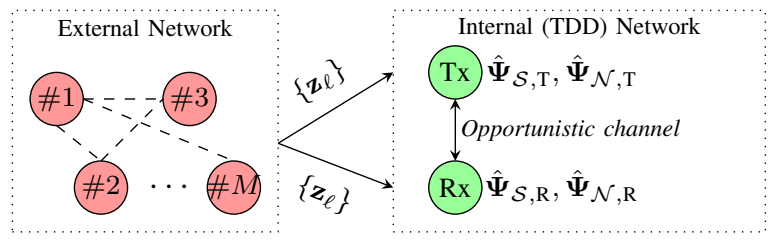

Fig. 1: Scenario-aware opportunistic communication in distributed networks.

We will focus our discussion on the scenario depicted in Fig. 1. The so-called external network is composed of $M$ users. Furthermore, these users may adopt different modulation and coding formats, with no prior or side information about them by the internal-network nodes. Indistinguishably, they exploit a fraction of $N$ degrees-of-freedom ${ }^{1}$ (DoF). Regarding to the internal network, we assume it is composed by a transmitter-receiver pair wishing to setup a communication link. The objective of internal-network users is to access the DoF sensed as available at each internal-network node and transmit information, working on TDD mode, providing non or little interference to external-network users.

It is worth pointing out that available and occupied DoF, encompassed in bases $\boldsymbol{\Psi}_{\mathcal{S}}$ and $\Psi_{\mathcal{N}}$, span orthogonal subspaces, further on referred as signal and noise subspaces, respectively. Therefore, internal-network users have to decide whether a DoF, namely $\zeta_{n}$, for $n=1, \ldots, N$, belongs to the signal or noise subspace. In order to do so, each internal-network user will sense the network conditions. Using local noisy observations $\mathbf{z}_{\ell}$ from the external network, they decide if a DoF $\zeta_{n}$ is available or occupied by solving a hypothesis test:

$$
\begin{aligned}
& \mathcal{H}_{0}: \zeta_{n} \text { is available } \\
& \mathcal{H}_{1}: \zeta_{n} \text { is occupied }
\end{aligned},
$$

for $n=1, \ldots, N$. How this hypothesis test or sensing problem is solved is out of the scope of this work. A broad literature

\footnotetext{
${ }^{1}$ In this work, we refer to degrees-of-freedom $(\mathrm{DoF})$ as done in [12], [13].
} 
may be found on spectrum sensing (cf. [14], [15] and references therein). Since internal-network nodes will only use local observations to solve (1), sensed signal and noise subspaces at each internal-network node will be, with high probability, slightly different.

\section{A. Scenario-Adapted Opportunistic Communication}

In order to avoid or mitigate interferences to externalnetwork users, internal transmitter and receiver should adapt or shape their signals in such a way that

$$
\boldsymbol{\Psi}_{\mathcal{S}}^{H} \varphi(\mathbf{r})=\mathbf{0}
$$

where $\varphi(\mathbf{r})$ is the pulse shaping filter designed at an arbitrary geographical position $\mathbf{r}$ (transmitter or receiver). However, due to sensing uncertainties ${ }^{2}$, the sensed signal-subspace basis at each geographical position will differ. In other words, letting $\hat{\mathbf{\Psi}}_{\mathcal{S}}(\mathbf{r})$ be the basis sensed at position $\mathbf{r}$, we may decompose this basis as follows:

$$
\hat{\boldsymbol{\Psi}}_{\mathcal{S}}(\mathbf{r})=\left[\begin{array}{l:l}
\tilde{\boldsymbol{\Psi}}_{\mathcal{S}}(\mathbf{r}) & \boldsymbol{\Xi}(\mathbf{r})]
\end{array}\right.
$$

where $\tilde{\boldsymbol{\Psi}}_{\mathcal{S}}(\mathbf{r})$ encompasses those DoF which are sensed as occupied and are actually occupied, whereas $\boldsymbol{\Xi}(\mathbf{r})$ contains those DoF which are available and are detected as occupied. Recalling (3), internal-network user at position $\mathbf{r}$ will design a waveform not only orthogonal to $\tilde{\Psi}_{\mathcal{S}}(\mathbf{r})$, but also orthogonal to $\Xi(\mathbf{r})$, incurring in a performance loss due to noise enhancement and energy loss.

In order to diminish these effects, pulse shaping filters $\varphi(\mathbf{r})$ should be designed to robustly exploit the sensed noise subspace and mitigate the performance loss, while avoiding cross-interferences between external and internal users. The design of such shaping filters is derived by the authors in [6], [16], [17]. In short, pulse shaping filters may be any column of the orthogonal projector onto the noise subspace, defined as $\hat{\mathbf{P}}_{\mathcal{N}}(\mathbf{r}) \triangleq \hat{\mathbf{\Psi}}_{\mathcal{N}}(\mathbf{r}) \hat{\mathbf{\Psi}}_{\mathcal{N}}^{H}(\mathbf{r})$. Therefore, internal-network nodes are able to obtain a waveform-book of $N$ elements. It is worth noting that the existence of $N$ different waveforms at each node may yield a detection uncertainty. Nevertheless, when internal nodes pre-agree a waveform selection criterion, waveforms can be detected. Contrarily, if there is a selection ambiguity or there is no a pre-agreed selection criterion, an internal receiving node must decide, without side information, which waveform has to be used to detect a signal from a neighboring node.

In any manner, although these $N$ waveforms have been designed accounting solely for local observations from external network, neighboring users will be able to detect them without side information on the noise subspace of their neighbors. Nonetheless, the waveform detectability requires the existence of some invariances. As reported in [6], designed waveforms present invariance to rotations within the effective noise subspace and invariance to subspace uncertainties.

\section{B. Effects of Sensing Uncertainties on Waveform Detection}

Recall that signal and noise subspaces are identified at each internal-network node by means of local observations from

\footnotetext{
${ }^{2}$ Sensing uncertainties refer to sensing errors caused by poor monitoring conditions (due to shadowing, multipath fading or outage of sensing channels) and by the miss-detection probability, inherent to all detection schemes.
}

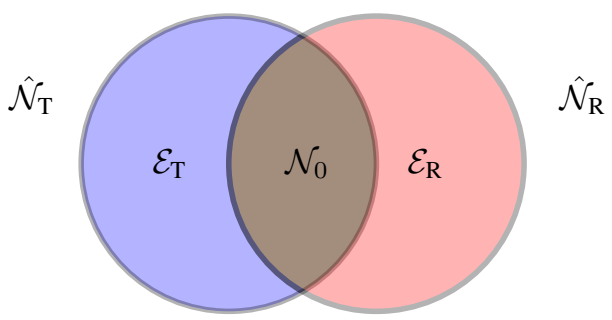

Fig. 2: Graphical representation of the relation between noise subspaces at internal transmitting and receiving nodes $\hat{\mathcal{N}}_{\mathrm{T}}$ and $\hat{\mathcal{N}}_{\mathrm{R}}$, respectively.

external network in a noncooperative fashion.

As a consequence, only a fraction of the DoF classified as available will be commonly detected as available at each internal-network node. Formally, let $\hat{\mathcal{N}}_{\mathrm{T}}$ and $\hat{\mathcal{N}}_{\mathrm{R}}$ be the sensed noise subspaces at internal transmitter and receiver, respectively. Notice that the size of these subspaces are $K_{\mathrm{T}}$ and $K_{\mathrm{R}}$, respectively. Therefore, we may define the effective noise subspace as follows:

$$
\mathcal{N}_{0}=\hat{\mathcal{N}}_{\mathrm{T}} \cap \hat{\mathcal{N}}_{\mathrm{R}}
$$

In other words, the effective noise subspace encompasses the DoF that are commonly detected as available at each internal node. Taking into account this rationale, we may write each noise subspace as (see Fig. 2 for clarity)

$$
\hat{\mathcal{N}}_{\mathrm{T}}=\mathcal{N}_{0} \cup \mathcal{E}_{\mathrm{T}} \text { and } \hat{\mathcal{N}}_{\mathrm{R}}=\mathcal{N}_{0} \cup \mathcal{E}_{\mathrm{R}}
$$

It is worth noting that $\mathcal{E}_{\mathrm{T}}$ and $\mathcal{E}_{\mathrm{R}}$ contains the DoF that are only detected as available at one internal-network node. Therefore, it is straightforward to see that $\mathcal{N}_{0} \cap \mathcal{E}_{\mathbf{r}}=\emptyset$ and $\mathcal{E}_{\mathrm{T}} \cap \mathcal{E}_{\mathrm{R}}=\emptyset$. Notice that only the power transmitted through $\mathcal{N}_{0}$ will be sensed by internal receiver, whereas the one transmitted through $\mathcal{E}_{\mathrm{T}}$ will be lost. Equivalently, receiver will only detect noise and/or interferences from $\mathcal{E}_{\mathrm{R}}$. Hence, by letting $S_{\mathrm{R}}$ and $N_{0}$ be the received power and the one-sided noise spectral density, the signal-to-noise ratio (SNR) at receiver is given by

$$
\mathrm{SNR}_{\mathrm{R}}=\frac{\left(1-\frac{\kappa_{\mathrm{T}}}{K_{\mathrm{T}}}\right) S_{\mathrm{R}}}{\left(1-\frac{\kappa_{\mathrm{R}}}{K_{0}}\right) N_{0}}=\Gamma_{\mathrm{unc}} \cdot \frac{S_{\mathrm{R}}}{N_{0}}=\Gamma_{\mathrm{unc}} \cdot \mathrm{SNR}_{0}
$$

where $\mathrm{SNR}_{0}$ is the effective SNR when there are no uncertainties, and $\Gamma_{\text {unc }}$ is a loss factor due to the uncertainties.

Finally, by making a slight abuse of notation, we can define the noise-subspace basis at geographical position $\mathbf{r}$, in the pairwise sense, as

$$
\hat{\boldsymbol{\Psi}}_{\mathcal{N}}(\mathbf{r})=\left[\begin{array}{l:l}
\boldsymbol{\Psi}_{\mathcal{N}}^{(0)} & \Delta(\mathbf{r})]
\end{array}\right.
$$

with $\Psi_{\mathcal{N}}^{(0)}$ spanning the effective noise subspace, and $\boldsymbol{\Delta}(\mathbf{r})$ encompassing those DoF that belongs to $\mathcal{E}_{\mathbf{r}}$. Notice that, by defining $K_{0}=\operatorname{dim}\left(\mathcal{N}_{0}\right)$ and $\kappa(\mathbf{r})=\operatorname{dim}\left(\mathcal{E}_{\mathbf{r}}\right)$, the number of available DoF at position $\mathbf{r}$ is given by $K(\mathbf{r})=K_{0}+\kappa(\mathbf{r})$.

\section{Problem Statement}

As we may conclude from (6), sensing uncertainties degrade the performance of opportunistic communications, due to a worse waveform detection. In this sense, this work addresses the distributed identification of the effective noise subspace at 
each internal-network user. In other words, the noise subspace is sensed at each internal node using only local observations from the wireless environment without end-to-end cooperation. We further on addressed how this nodes may select those DoF belonging to the effective noise subspace, whereas the remaining ones are discarded to diminish the effects of uncertainties.

\section{WAVEForm Detection And EFFECTIVE Noise SUBSPACE IDENTIFICATION}

In the ideal scenario where there are not sensing uncertainties, i.e. $\kappa_{\mathrm{T}}=\kappa_{\mathrm{R}}=0$, we note that $\hat{\mathcal{N}}_{\mathrm{T}}=\hat{\mathcal{N}}_{\mathrm{R}}$. Thus, the waveform detection problem reduces to decide which of the $N$ waveforms has been transmitted to use it as a matched filter.

Nevertheless, whenever $\left\{\kappa_{\mathrm{T}}, \kappa_{\mathrm{R}}\right\} \neq 0$, matched-filter detection is no longer optimal. The authors introduce in [6] an $\ell_{1}$-norm scheme that prunes the noise subspace at receiver and ideally detects only on the intersection $\hat{\mathcal{N}}_{0}$ without prior knowledge or side information about the noise subspace sensed at transmitter side. In other words, receiver tries to identify the effective noise subspace, design an orthogonal projection onto $\mathcal{N}_{0}$, and select a waveform $\tilde{\varphi}$ belonging exclusively to the estimated $\mathcal{N}_{0}$. Based on this scheme, we will present in this paper a two-step subspace agreement methodology, with and without side information.

Let $\mathbf{x}_{m}$ be the signal at receiver's input when the symbol $a[m]$ has been transmitted. The signal $\mathbf{x}_{m}$ is given by

$$
\mathbf{x}_{m}=a[m] \sqrt{S_{\mathrm{R}}} \boldsymbol{\varphi}_{\mathrm{T}, k}+\boldsymbol{v}_{1}+\boldsymbol{v}_{2}
$$

where $\boldsymbol{\varphi}_{\mathrm{T}, k}$ is the $k$-th element from the transmitter's waveform-book, $\boldsymbol{v}_{1}$ is a complex zero-mean white Gaussian noise from $\mathcal{N}_{0}$, and $\boldsymbol{v}_{2}$ is a complex noise from $\mathcal{E}_{\mathrm{R}}$. Generally, $\boldsymbol{v}_{2}$ will not be zero-mean nor white, in order to account for interferences. Internal receiving node may estimate (8) as

$$
\hat{\mathbf{x}}=\tilde{\mathbf{P}}_{\mathcal{N}, \mathrm{R}} \boldsymbol{\alpha}
$$

where $\boldsymbol{\alpha} \in\{0,1\}^{N}$ selects the appropriate column from the modified orthogonal projector $\tilde{\mathbf{P}}_{\mathcal{N}, \mathrm{R}}$, defined as

$$
\tilde{\mathbf{P}}_{\mathcal{N}, \mathrm{R}}=\sum_{i=1}^{K_{\mathrm{R}}} \lambda_{i} \mathbf{P}_{i}^{(\mathrm{R})}
$$

being $\mathbf{P}_{i}^{(\mathrm{R})}$ a rank-one orthogonal projector onto a receiver's noise subspace singleton, and parameter $\lambda_{i} \in\{0,1\}$ controls if the $i$-th singleton belongs to the intersection or not.

By defining $\boldsymbol{\beta}=\boldsymbol{\lambda} \otimes \boldsymbol{\alpha}$, with $\otimes$ being the Kronecker product, the estimation problem in (9) can be jointly addressed as

$$
\hat{\boldsymbol{\beta}}=\underset{\boldsymbol{\beta}}{\arg \min }\|\boldsymbol{\beta}\|_{1} \text { s.t. } \mathbf{x}=\mathbf{P} \boldsymbol{\beta}
$$

with $\boldsymbol{\lambda}=\left[\lambda_{1}, \ldots, \lambda_{K_{\mathrm{R}}}\right]^{T}$ and $\mathbf{P}=\left[\mathbf{P}_{1} \cdots \mathbf{P}_{K_{\mathrm{R}}}\right] \in \mathbb{C}^{N \times N K_{\mathrm{R}}}$. It is worth noting that basis pursuit (BP) algorithm exhibits noise sensitivity. Therefore, as explained in [6], instead of considering just one observation from the transmitter, receiver may obtain $Q$ observations. These $Q$ observations are then stacked in the extended data vector $\tilde{\mathbf{x}}=\left[\mathbf{x}_{1}^{T} \cdots \mathbf{x}_{Q}^{T}\right]^{T}$. Hence, by defining the matrix $\boldsymbol{\Phi} \in \mathbb{C}^{N Q \times N K_{\mathrm{R}}}$, such that $\boldsymbol{\Phi}=$ $\left[\mathbf{P}^{T} \ldots \mathbf{P}^{T}\right]^{T}$, (11) can be cast as

$$
\hat{\boldsymbol{\beta}}=\underset{\boldsymbol{\beta}}{\arg \min }\|\boldsymbol{\beta}\|_{1} \text { s.t. }\|\tilde{\mathbf{x}}-\boldsymbol{\Phi} \boldsymbol{\beta}\|_{2}^{2} \leq \epsilon^{2} .
$$

It is worth noting that the computational complexity in (12) has increased. It is straightforward to show the equivalence between (12) and

$$
\hat{\boldsymbol{\beta}}=\underset{\boldsymbol{\beta}}{\arg \min }\|\boldsymbol{\beta}\|_{1} \text { s.t. } \sum_{q=1}^{Q}\left\|\mathbf{x}_{q}-\mathbf{P} \boldsymbol{\beta}\right\|_{2}^{2} \leq \epsilon^{2},
$$

i.e. the error of the considered $N Q$-length data block $\tilde{\mathbf{x}}$ is equal to the cumulative error of each sub-block $\mathbf{x}_{q}$ of length $N$. It is worth noting that the detection scheme in (13) is selecting a column of the orthogonal projector onto the estimated intersection of noise subspaces. Hence, the effects of sensing uncertainties at receiver are diminished.

\section{Feedback in Distributed Subspace Agreement}

In the previous Section, we have seen how internal receiving node may estimate the effective noise subspace. The question is how the transmitter may also identify it, in order not to lose energy nor interfere external-network communication. Herein, we present two different approaches to achieve consensus.

\section{A. Noncooperative Subspace Agreement}

It is worth noting that the pulse shaping filter used by the receiver to detect internal transmitted signal is given by $\tilde{\boldsymbol{\varphi}}_{\mathrm{R}}=\mathbf{P} \hat{\boldsymbol{\beta}}$, where $\mathbf{P}$ is defined in (11), and $\hat{\boldsymbol{\beta}}$ is the solution to (13). Therefore, internal receiver use this pulse shaping filter to improve the communication with internal transmitter.

Since internal-network nodes are operating in TDD mode, internal transmitting node may also try to detect $\mathcal{N}_{0}$. To do so, internal transmitter should follow the same procedure performed by internal receiver. Recall how receiver estimates transmitter's waveform (9). To select which DoF belongs to $\mathcal{N}_{0}$, the problem is analogous to (9), but using

$$
\tilde{\mathbf{P}}_{\mathcal{N}, \mathrm{T}}=\sum_{i=1}^{K_{\mathrm{T}}} \pi_{i} \mathbf{P}_{i}^{(\mathrm{T})}
$$

where $\mathbf{P}_{i}^{(\mathrm{T})}$ is a rank-one orthogonal projector onto a transmitters's noise subspace singleton and $\pi_{i}$ plays the role of $\lambda_{i}$, instead of $\tilde{\mathbf{P}}_{\mathcal{N}, \mathrm{R}}$. Therefore, by taking into consideration the ideas presented in Sec. III, the agreement problem can be finally cast as in (13).

As we will see in Sec. $\mathrm{V}$, this noncooperative scheme presents a very good performance when the working conditions are appropriate, i.e. high pulse energy-to-noise ratio $\frac{E_{p}}{N_{0}}$ and large data block-length $Q$. However, whether it is no possible to operate in these regimes, internal-network users will iteratively reduce their noise subspaces until achieving the intersection $\mathcal{N}_{0}$. The convergence of this iterative subspace pruning depends on the operating conditions and opportunistic channel state. In the next Subsection, we will introduce a cooperative scheme in which receiver helps transmitter by providing some side information about the estimated $\mathcal{N}_{0}$ by internal receiving node. 


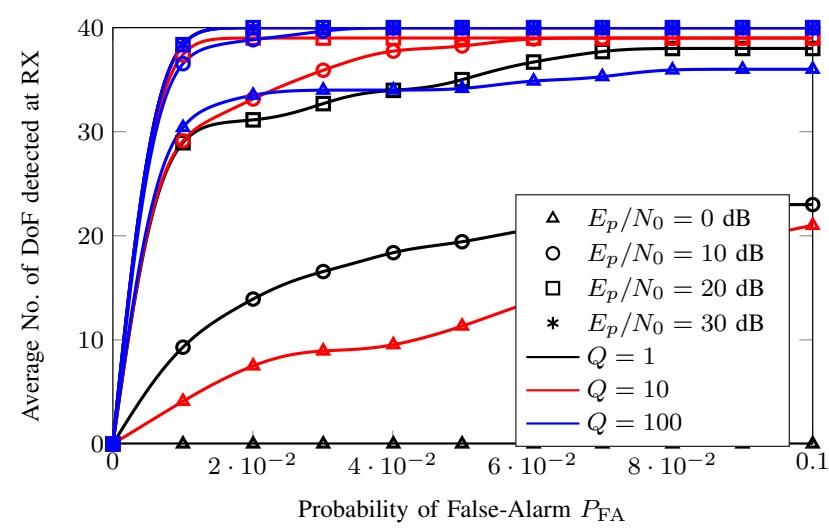

Fig. 3: Avg. no. of detected DoF at RX for $E_{p} / N_{0}=\{0,10,20,30\} \mathrm{dB}$ and block-length $Q=\{1,10,100\}$.

\section{B. Cooperative Subspace Agreement}

In the concurrence scheme presented so far, internal receiver does not help internal transmitter to estimate $\mathcal{N}_{0}$. Nevertheless, we note that whether receiver provides some side information on the estimated effective noise subspace $\hat{\mathcal{N}}_{0}$ to the transmitter, consensus may be easier to achieve.

First of all, notice that vector $\boldsymbol{\beta} \in\{0,1\}^{N K_{\mathrm{R}}}$ can, in fact, be decomposed as a (sparse) $K_{\mathrm{R}} \times N$ matrix $\mathbf{B}$. Let $[\mathbf{B}]_{i j}$ be the $i j$-th entry of $\mathbf{B}$. Because only one column of the orthogonal projector will be selected, notice that

$$
[\mathbf{B}]_{i j}=\left\{\begin{array}{cl}
\hat{\lambda}_{i} & \text { if } j \text { is selected } \\
0 & \text { otherwise }
\end{array} .\right.
$$

Hence, internal receiver may estimate the dimensions of the estimated effective noise subspace as

$$
\hat{K}_{0}=\|\hat{\boldsymbol{\lambda}}\|_{0} \cong\|\hat{\boldsymbol{\beta}}\|_{0}
$$

with $\|\cdot\|_{0}$ standing for the $\ell_{0}$-norm (i.e. the number of nonzero elements). With this information, internal receiving node can construct a feedback message $\mathbf{f}$ such that

$$
\mathbf{f}=\left[\hat{K}_{0}, \tilde{\varphi}_{\mathrm{R}}^{T}\right]^{T} \in \mathbb{C}^{N+1} .
$$

The side information encompassed in (17) can be exploited by internal transmitting node as follows. It is worth noting that the BP optimization proposed in Sec. IV-A can be seen as a sparse recovery problem without information on the required sparsity. Nevertheless, thanks to the feedback message in (17), internal transmitter is now aware of the required sparsity level. Therefore, by letting $\gamma$ be the analogous of $\boldsymbol{\beta}$ at transmitter side, a more informative problem can be tackled. In this sense, we propose the following optimization:

$$
\min _{\gamma}\left\|\mathbf{P} \boldsymbol{\gamma}-\tilde{\varphi}_{\mathrm{R}}\right\|_{2}^{2} \text { s.t. }\|\boldsymbol{\gamma}\|_{0} \leq \tilde{K}_{0},
$$

where $\tilde{\varphi}_{\mathrm{R}}$ and $\tilde{K}_{0}$ are provided by transmitter through the feedback message in (17). Unfortunately, the sparsity constraint in (18) is an NP-hard (i.e. non-convex) problem. A classical approach in compressed sensing and sparse recovery literature consists in relaxing this constraint such that

$$
\min _{\gamma}\left\|\mathbf{P} \gamma-\tilde{\varphi}_{\mathrm{R}}\right\|_{2}^{2} \text { s.t. }\|\gamma\|_{1} \leq \tilde{K}_{0} .
$$

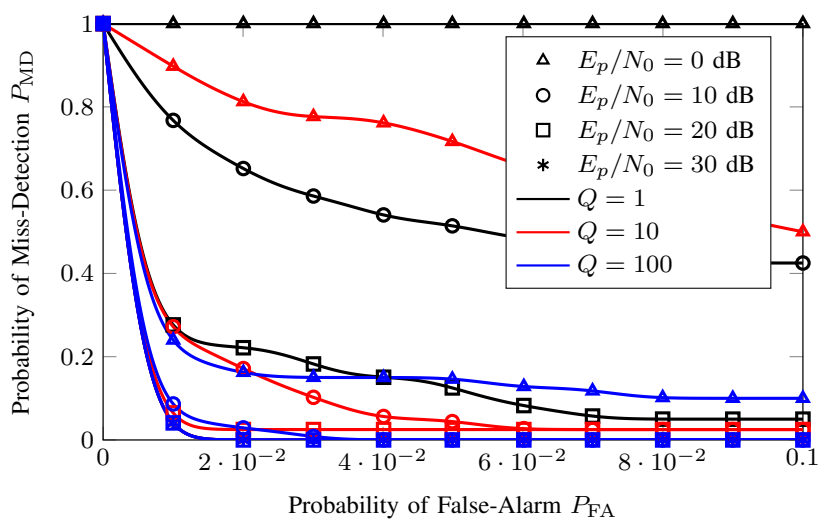

Fig. 4: Complementary ROC of internal transmitting node using the noncooperative subspace consensus scheme averaged over $10^{4}$ independent realizations.

It is worth noting that the optimization problem tackled in (19) is the well-known basis pursuit denoising (BPDN), also known as least absolute shrinkage and selection operator (LASSO).

Although the exact recovery cannot be guaranteed in general, as stated in Lemma 1 (proved in Appendix), (18) and (19) can be equivalent, and hence the sparse vector $\gamma$ can be recovered.

Lemma 1. Optimization problem in (19) is equivalent to that in (18), under the Restricted Isometry Property (RIP) [18], when nodes work with canonical basis or in the large-DoF regime. Otherwise, the equivalence cannot be guaranteed.

\section{Simulation Analysis}

In this Section, we provide a numerical analysis of the proposed distributed consensus schemes. We consider an ambient space of $N=64 \mathrm{DoF}$. A total of $M$ external users occupy $D=24$ DoF. Hence, there is a 40-dimensional subspace available for opportunistic communication. Taking sensing uncertainties into account, both transmitter and receiver overestimate the noise subspace, with absolute uncertainties of $\kappa_{\mathrm{T}}=\kappa_{\mathrm{R}}=12 \mathrm{DoF}$. Note that performance of proposed consensus schemes depends on the operating point of internal receiving node. Thus, we have depicted in Fig. 3 the number of DoF detected as available at RX. Due to the favorable operating conditions, internal receiver is able to perfectly detect $\mathcal{N}_{0}$ for relatively small Probability of False-Alarm $\left(P_{\mathrm{FA}}\right)$. In the sequel, we are going to consider a particular operating point in which receiver has perfectly detected $\mathcal{N}_{0}$.

Complementary Receiver Operating Characteristics (ROC) is depicted in Figs. 4 and 5 for the noncooperative and cooperative schemes, respectively. To minimize the probability of miss-detection $P_{\mathrm{MD}}$, we have considered a Neyman-Pearson threshold. Notice that both agreement schemes present a very good performance under the considered operating conditions for $P_{\mathrm{FA}}$ lower than 0.1. Nevertheless, since the cooperative scheme has side information about the estimated $\mathcal{N}_{0}$ at receiver, it is able to work at much lower $P_{\mathrm{FA}}$ than the noncooperative.

In Fig. 6, we have depicted the number of DoF detected at internal transmitter when noncooperative (solid) and cooperative (dashed) consensus schemes are considered. Notice that, albeit both schemes detect the whole intersection for very small $P_{\mathrm{FA}}$, cooperative scheme exhibits a better performance in demanding 


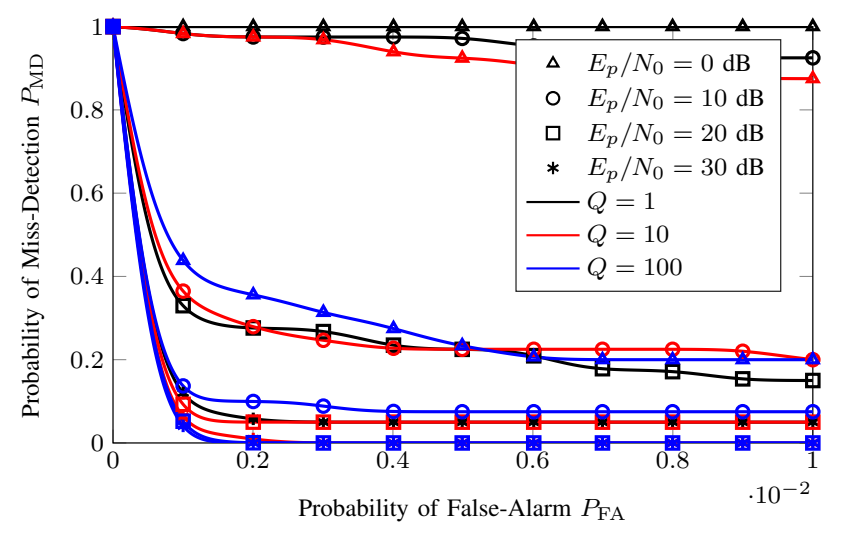

Fig. 5: Complementary ROC of internal transmitting node using the cooperative subspace consensus scheme averaged over $10^{4}$ independent realizations.

scenarios (low $E_{p} / N_{0}$ and small $Q$ ) than the noncooperative one. This crucial aspect is of paramount importance because internal network users will be able to discard the uncommon DoF ideally canceling the effects of sensing uncertainties.

\section{CONCLUSIONS}

This paper has addressed the distributed subspace consensus in opportunistic communications. Because internalnetwork users accounts solely for local observations of the environment, sensing uncertainties may degrade the overall system performance. Two distributed subspace consensus have been presented when internal transmitting and receiving nodes cooperate or not. As a result, they will ideally use the same subspace reducing hence the performance loss. Simulation analysis is provided to corroborate theoretical results.

\section{APPENDIX}

In this appendix, we sketch the proof of Lemma 1. A measurement matrix $\Omega$ satisfies the RIP for all $n$-sparse vectors $\boldsymbol{v}$ with parameters $(n, \varepsilon)$ whether

$$
(1-\varepsilon)\|\boldsymbol{v}\|_{2} \leq\|\boldsymbol{\Omega} \boldsymbol{v}\|_{2} \leq(1+\varepsilon)\|\boldsymbol{v}\|_{2}, \forall \varepsilon \in(0,1)
$$

Recall that, in our problem, $\boldsymbol{v}=\gamma \in\{0,1\}^{N K_{\mathrm{T}}}$ and $\boldsymbol{\Omega}=\mathbf{P} \in$ $\mathbb{C}^{N \times N K_{\mathrm{T}}}$. Therefore, it is worth noting that

$$
\begin{aligned}
\|\gamma\|_{2} & =\sqrt{\gamma^{H} \gamma}=\sqrt{\hat{K}_{0}^{(\mathrm{T})}} \\
\|\mathbf{P} \gamma\|_{2} & =\sqrt{\sum_{i=1}^{K_{\mathrm{T}}} \lambda_{i}^{2}\left[\mathbf{P}_{i}\right]_{\hat{\iota}, \hat{\iota}} .}
\end{aligned}
$$

Notice that (21b) is equal to $\sqrt{\hat{K}_{0}^{(\mathrm{T})}}$ when the diagonal entries of each $\mathbf{P}_{i}$ are ones. By noting that this happens when rankone projectors are obtained from canonical basis or elements of bases are complex exponentials (which will asymptotically occur), RIP is satisfied in these two cases for all possible $\varepsilon$. Otherwise, the second inequality in (20) is always satisfied, but nothing can be said about the first one.

\section{REFERENCES}

[1] L. Lu, G. Y. Li, A. Maaref, and R. Yao, "Opportunistic transmission exploiting frequency- and spatial-domain degrees of freedom," IEEE Wireless Commun., vol. 21, no. 2, pp. 91-97, April 2014.

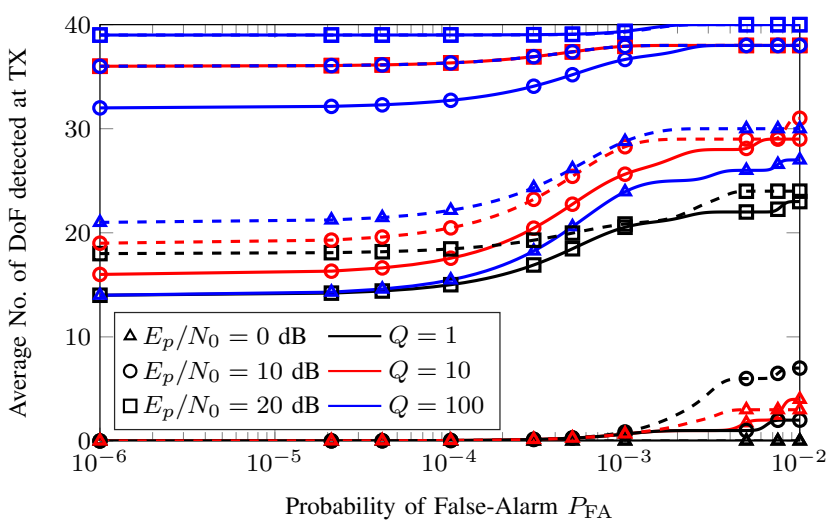

Fig. 6: Avg. no. of detected DoF at TX versus $P_{\text {FA }}$. Solid and dashed lines refers to noncooperative and cooperative consensus schemes.

[2] W. Liang, S. X. Ng, and L. Hanzo, "Cooperative overlay spectrum access in cognitive radio networks," IEEE Commun. Surveys Tut., vol. 19, no. 3, pp. 1924-1944, thirdquarter 2017.

[3] Y. Ma, Y. Gao, Y. Liang, and S. Cui, "Reliable and efficient sub-nyquist wideband spectrum sensing in cooperative cognitive radio networks," IEEE J. Sel. Areas Commun., vol. 34, no. 10, pp. 2750-2762, Oct 2016.

[4] M. Nguyen, N. Nguyen, D. B. Da Costa, H. Nguyen, and R. T. De Sousa, "Secure cooperative half-duplex cognitive radio networks with $k$-th best relay selection," IEEE Access, vol. 5, pp. 6678-6687, 2017.

[5] S. S. Kalamkar, J. P. Jeyaraj, A. Banerjee, and K. Rajawat, "Resource allocation and fairness in wireless powered cooperative cognitive radio networks," IEEE Trans. Commun., vol. 64, no. 8, pp. 3246-3261, Aug 2016.

[6] J. Borràs and G. Vázquez, "Decentralized shaping for pilot generation and detection in opportunistic communications," in 2019 IEEE Int. Conf. Commun. (ICC) [Accepted], May 2019.

[7] S. Kar and J. M. F. Moura, "Distributed consensus algorithms in sensor networks: Quantized data and random link failures," IEEE Trans. Signal Process., vol. 58, no. 3, pp. 1383-1400, March 2010.

[8] W. Ben-Ameur, P. Bianchi, and J. Jakubowicz, "Robust distributed consensus using total variation," IEEE Trans. Autom. Control, vol. 61, no. 6, pp. $1550-1564$, June 2016.

[9] B. Mishra, H. Kasai, P. Jawanpuria, and A. Saroop, "A riemannian gossip approach to subspace learning on grassmann manifold," Machine Learning, Jan 2019.

[10] F. Penna and S. Stanczak, "Decentralized eigenvalue algorithms for distributed signal detection in wireless networks," IEEE Trans. Signal Process., vol. 63, no. 2, pp. 427-440, Jan 2015.

[11] G. Li, T. Wimalajeewa, and P. K. Varshney, "Decentralized and collaborative subspace pursuit: A communication-efficient algorithm for joint sparsity pattern recovery with sensor networks," IEEE Trans. Signal Process., vol. 64, no. 3, pp. 556-566, Feb 2016.

[12] R. G. Gallager, Information Theory and Reliable Communications. John Wiley \& Sons, 1968.

[13] D. Tse and P. Viswanath, Fundamentals of Wireless Communication. Cambridge University Press, 2005.

[14] J. Font-Segura, G. Vázquez, and J. Riba, "Single and multi-frequency wideband spectrum sensing with side-information," IET Signal Process., vol. 8, no. 8, pp. 831-843, 2014.

[15] A. Ali and W. Hamouda, "Advances on spectrum sensing for cognitive radio networks: Theory and applications," IEEE Commun. Surveys Tut., vol. 19, no. 2, pp. 1277-1304, Secondquarter 2017.

[16] J. Borràs, J. Font-Segura, J. Riba, and G. Vázquez, "Dimension spreading for coherent opportunistic communications," in 2017 51 st Asilomar Conf. Signals, Syst. Comput., Oct 2017, pp. 1940-1944.

[17] J. Borràs and G. Vázquez, "Uncoordinated space-frequency pilot design for multi-antenna wideband opportunistic communications," in 2018 IEEE 19th Int. Workshop Signal Process. Adv. Wireless Commun. (SPAWC), Jun 2018, pp. 1-5.

[18] E. J. Candes and T. Tao, "Decoding by linear programming," IEEE Trans. Inf. Theory, vol. 51, no. 12, pp. 4203-4215, Dec 2005. 